\title{
REGULAR BASES AT NON-ISOLATED POINTS AND METRIZATION THEOREMS
}

\author{
FUCAI LIN, SHOU LIN, AND HEIKKI J. K. JUNNILA
}

\begin{abstract}
In this paper, we define the spaces with a regular base at non-isolated points and discuss some metrization theorems. We firstly show that a space $X$ is a metrizable space, if and only if $X$ is a regular space with a $\sigma$-locally finite base at nonisolated points, if and only if $X$ is a perfect space with a regular base at non-isolated points, if and only if $X$ is a $\beta$-space with a regular base at non-isolated points. In addition, we also discuss the relations between the spaces with a regular base at nonisolated points and some generalized metrizable spaces. Finally, we give an affirmative answer for a question posed by F. C. Lin and S. Lin in [7, which also shows that a space with a regular base at non-isolated points has a point-countable base.
\end{abstract}

\section{INTRODUCTION}

The bases of topological spaces occupy a core position in the study of the topological theories and metrization problems, which has produced many kinds of metrization theorems, and establishes a foundation for the topological development [12]. For example, the following is a classic metrization theorem.

Theorem 1.1. The following are equivalent for a space $X$ :

(1) $X$ is metrizable;

(2) $X$ is a $T_{1}$-space with a regular base;

(3) $X$ is a regular space with a $\sigma$-locally finite base.

In recent years, the theory of regular bases in topological spaces played an important role in generalized metrizable spaces [2, 17. On the other hand, in the study of the theories of topological spaces, we are mainly concerned with the properties of neighborhoods on non-isolated points, and also discuss the relation between their properties and global properties. For example, a study of spaces with a sharp base, a weakly uniform base or an uniform base at non-isolated points [2, 3, 7] shows that some properties of a non-isolated point set of a topological space will help us discuss the global construction of a space. Especially, a space $X$ with a uniform base at non-isolated points if and only if $X$ is the open and boundary-compact image of a metric space [7]. The most typical example is the spaces obtained from a metrizable space by isolating the points of a subset.

Let $\mathcal{B}$ be a base for a space $X$. For any $x \in X$, the base $\mathcal{B}$ of $X$ is called regular at a point $x$ if, for every neighborhood $U$ of $x$, there exists an open subset $V$ such that $x \in V \subset U$ and $\{B \in \mathcal{B}: B \cap V \neq \emptyset$ and $B \not \subset U\}$ is finite.

2000 Mathematics Subject Classification. 54D70; 54E35; 54D20.

Key words and phrases. Metrization; regular bases; locally-finite families; $\beta$-spaces; proto-metrizable; discretization; point-regular bases.

Supported by the NSFC(No. 10971185) and the Educational Department of Fujian Province(No. JA09166). 
By Theorem 1.1, every metric space has a base which is regular at non-isolated points. However, there exists a non-metrizable space with a base which is regular at non-isolated points, see the following Example 1.2

Example 1.2. Let $X$ be the closed unit interval $\mathbb{I}=[0,1]$ and $B$ a Bernstein subset of $I$. In other words, $B$ is an uncountable set which contains no uncountable closed subset of $I$. Endow $X$ with the following topology, i.e., Michael line [15]: $G$ is an open subset for $X$ if and only if $G=U \cup Z$, where $U$ is an open subset of $\mathbb{I}$ with Euclidean topology and $Z \subset B$. Let $\mathcal{B}$ be a base of $\mathbb{I}$ with the Euclidean topology, where $\mathcal{B}$ is regular at every point of $\mathbb{I}$. Then $\mathcal{P}=\mathcal{B} \cup\{\{x\}: x \in B\}$ is a base for $X$ and also regular at non-isolated points.

Hence this causes our interests in a study of spaces with a base which is regular at non-isolated points, and the related problems of the metrizability. In this paper, we shall prove that spaces with a regular base at non-isolated points are strictly between the discretizations of metrizable spaces and proto-metrizable spaces, and we also obtain some metrization theorems which help us to better understand the relation between the properties at non-isolated points and global properties in the study the generalized metrizable spaces.

In this paper all spaces are $T_{1}$ unless it is explicitly stated which separation axiom is assumed, and all maps are continuous and onto. By $\mathbb{R}, \mathbb{N}$, denote the set of real numbers and positive integers, respectively. For a space $X$, let $I=I(X)=\{x$ : $x$ is an isolated point of $X\}$ and $\mathcal{I}(X)=\{\{x\}: x \in I(X)\}$. Let $\mathcal{P}$ be a family of subsets for $X$, and we denote

$$
\begin{aligned}
& \operatorname{st}(x, \mathcal{P})=\cup\{P \in \mathcal{P}: x \in P\}, x \in X ; \\
& \operatorname{st}(A, \mathcal{P})=\cup\{P \in \mathcal{P}: A \cap P \neq \emptyset\}, A \subset X ; \\
& \mathcal{P}^{m}=\{P \in \mathcal{P}: \text { if } P \subset Q \in \mathcal{P}, \text { then } Q=P\} .
\end{aligned}
$$

Readers may refer to [6, 13, for unstated definitions and terminology.

\section{Regular Bases at nON-ISOlated POINTS}

Definition 2.1. Let $\mathcal{B}$ be a base of a space $X . \mathcal{B}$ is a regular base, see e.g. [6] (regular base at non-isolated points, resp.) for $X$ if for each (non-isolated, resp.) point $x \in X$, $\mathcal{B}$ is regular at $x$.

It is obvious that regular bases $\Rightarrow$ regular bases at non-isolated points, but regular bases at non-isolated points $\nRightarrow$ regular bases by Example 1.2 .

Definition 2.2. Let $\left\{\mathcal{W}_{i}\right\}_{i \in \mathbb{N}}$ be a sequence of open covers of a space $X$ and $\mathcal{I}(X) \subset$ $\bigcup_{i \in \mathbb{N}} \mathcal{W}_{i} . \quad\left\{\mathcal{W}_{i}\right\}_{i \in \mathbb{N}}$ is called a strong development, see e.g. [6] (strong development at non-isolated points, resp.) for $X$ if for every $x \in X(x \in X-I)$ and each neighborhood $U$ of $x$ there exist a neighborhood $V$ of $x$ and an $i \in \mathbb{N}$ such that $\operatorname{st}\left(V, \mathcal{W}_{i}\right) \subset U$. If $\left\{\mathcal{W}_{i}\right\}_{i \in \mathbb{N}}$ is a strong development at non-isolated points, then so is $\left\{\mathcal{W}_{i} \cup \mathcal{I}(X)\right\}_{i \in \mathbb{N}}$.

The following Lemma 2.3 is proved similarly to Lemma 5.4 .3 in [6], and leave to the reader the easy proofs of Lemma 2.4 and 2.5 .

Lemma 2.3. If $\mathcal{B}$ is a regular base at non-isolated points for a space $X$, then the family $\mathcal{B}^{m} \subset \mathcal{B}$ is locally finite at non-isolated points and also covers $X-I$. 
Lemma 2.4. Let $\mathcal{B}$ be a regular base at non-isolated points for $X$. If $\mathcal{B}^{\prime} \subset \mathcal{B}$ is pointfinite at non-isolated points, then $\mathcal{B}^{\prime \prime}=\left(\mathcal{B}-\mathcal{B}^{\prime}\right) \cup \mathcal{I}(X)$ is a regular base at non-isolated points for $X$.

Lemma 2.5. If $\mathcal{B}$ is a regular base at non-isolated points for $X$, put

$$
\mathcal{B}_{1}=\mathcal{B}^{m}, \quad \mathcal{B}_{i}=\left[\left(\mathcal{B}-\bigcup_{j=1}^{i-1} \mathcal{B}_{j}\right) \cup \mathcal{I}(X)\right]^{m}, i=2,3, \cdots .
$$

Then $\mathcal{B}=\left(\bigcup_{i=1}^{\infty} \mathcal{B}_{i}\right) \cup \mathcal{I}(X)$, and for each $i \in \mathbb{N}, \mathcal{B}_{i}$ is locally finite at non-isolated points and $\mathcal{B}_{i+1} \cup \mathcal{I}(X)$ refines $\mathcal{B}_{i} \cup \mathcal{I}(X)$.

Recall that a topological space $X$ is monotonically normal [10] if for each ordered pair $(p, C)$, where $C$ is a closed set for $X$ and $p \in X-C$, there exists an open subset $H(p, C)$ satisfying the following conditions:

(i) $p \in H(p, C) \subset X-C$;

(ii) For every closed subset $D$ for $X$, if $D \subset C$, then $H(p, C) \subset H(p, D)$;

(iii) If $p \neq q \in X$, then $H(p,\{q\}) \cap H(q,\{p\})=\emptyset$.

$\mathrm{A} \mathrm{T}_{2}$-paracompact space or monotonically normal space is a collectionwise normal space [10].

Lemma 2.6. If a space $X$ has a strong development at non-isolated points, then $X$ is a monotonically normal and paracompact space.

Proof. Let $\left\{\mathcal{W}_{i}\right\}_{i \in \mathbb{N}}$ be a strong development at non-isolated points for $X$, where $\mathcal{W}_{i+1}$ refines $\mathcal{W}_{i}$ for every $i \in \mathbb{N}$.

(1) Claim. Let $A$ be a closed subset for $X$. If $x \in(X-A) \cap(X-I)$, then there exists an $i \in \mathbb{N}$ such that $\operatorname{st}\left(x, \mathcal{W}_{i}\right) \cap \operatorname{st}\left(A, \mathcal{W}_{i}\right)=\emptyset$.

In fact, since $X-A$ is an open neighborhood of $x$, there exists a $j \in \mathbb{N}$ and an open neighborhood $V$ of $x$ such that $\operatorname{st}\left(V, \mathcal{W}_{j}\right) \subset X-A$. Also, there exists a $i \geq j$ such that $\operatorname{st}\left(x, \mathcal{W}_{i}\right) \subset V$. Since $\operatorname{st}\left(A, \mathcal{W}_{i}\right) \subset X-V$, we have $\operatorname{st}\left(x, \mathcal{W}_{i}\right) \cap \operatorname{st}\left(A, \mathcal{W}_{i}\right)=\emptyset$.

(2) $X$ is a monotonically normal space.

Let $C$ be a closed subset for $X$ and $p \in X-C$. If $p \in I$, then we let $H(p, C)=\{p\}$; if $p \in X-I$, then there exists a minimum $n \in \mathbb{N}$ such that $\operatorname{st}\left(p, \mathcal{W}_{n}\right) \cap \operatorname{st}\left(C, \mathcal{W}_{n}\right)=\emptyset$ by (1), so we let $H(p, C)=\operatorname{st}\left(p, \mathcal{W}_{n}\right)$. Then $H(p, C)$ is an open subset for $X$. Clearly this definition of $H(p, C)$ satisfies the conditions (i) and (ii) in the above definition of monotonically normal spaces. We next prove that it also satisfies (iii). In fact, for any distinct points $p, q$ in $X-I$, fix the $n, m$ for which:

$$
H(p,\{q\})=\operatorname{st}\left(p, \mathcal{W}_{n}\right) \text { and } H(q,\{p\})=\operatorname{st}\left(q, \mathcal{W}_{m}\right) .
$$

Then

$$
\operatorname{st}\left(p, \mathcal{W}_{n}\right) \cap \operatorname{st}\left(q, \mathcal{W}_{n}\right)=\emptyset \text { and } \operatorname{st}\left(p, \mathcal{W}_{m}\right) \cap \operatorname{st}\left(q, \mathcal{W}_{m}\right)=\emptyset .
$$

By the choice of $n, m$, we have $n=m$, i.e, $H(p,\{q\}) \cap H(q,\{p\})=\emptyset$. Hence it also satisfies (iii) in the definition of monotonically normal spaces.

(3) $X$ is a paracompact space.

Let $\left\{G_{s}\right\}_{s \in S}$ be an open cover for $X$ and $S_{0}=\left\{s \in S: G_{s} \cap(X-I) \neq \emptyset\right\}$. Fix a well-order by " $<$ " on $S_{0}$. For every $i \in \mathbb{N}, s \in S_{0}$, put 
then $F_{s, i} \subset G_{s}$.

$$
F_{s, i}=X-\left(\operatorname{st}\left(X-G_{s}, \mathcal{W}_{i}\right) \cup\left(\bigcup_{s^{\prime}<s} G_{s^{\prime}}\right)\right),
$$

(3.1) The closed family $\left\{F_{s, i}\right\}_{s \in S_{0}, i \in \mathbb{N}}$ covers $X-I$.

Indeed, for every $x \in X-I$, there exists a minimum $s(x) \in S_{0}$ such that $x \in G_{s(x)}$. Since $\left\{\mathcal{W}_{i}\right\}_{i \in \mathbb{N}}$ is a strong development at non-isolated points for $X$, there exists an $i(x) \in \mathbb{N}$ such that $\operatorname{st}\left(x, \mathcal{W}_{i(x)}\right) \subset G_{s(x)}$. Hence $x \in F_{s(x), i(x)}$.

(3.2) For every $i \in \mathbb{N},\left\{F_{s, i}\right\}_{s \in S_{0}}$ is a discrete and closed family for $X$.

The family $\left\{F_{s, i}\right\}_{s \in S_{0}}$ is disjoint by construction, hence if $x \in I$ then $\{x\}$ is a neighborhood that intersects $F_{s, i}$ for at most one $s$. If $x \in X \backslash I$ then, using (3.1), $x \in \bigcup_{s \in S_{0}} G_{s}$. Hence there exists a minimum $s(x) \in S_{0}$ such that $x \in G_{s(x)}$. Then $G_{s(x)} \cap \operatorname{st}\left(x, \mathcal{W}_{i}\right)$ is an open neighborhood of $x$. If $s^{\prime}<s(x)$, then $x \in X-G_{s^{\prime}}$, so we have

$$
\operatorname{st}\left(x, \mathcal{W}_{i}\right) \subset \operatorname{st}\left(X-G_{s^{\prime}}, \mathcal{W}_{i}\right) \text { and } \operatorname{st}\left(x, \mathcal{W}_{i}\right) \cap F_{s^{\prime}, i}=\emptyset ;
$$

If $s^{\prime}>s(x)$, then $G_{s(x)} \cap F_{s^{\prime}, i}=\emptyset$, so there is only one member of $\left\{F_{s, i}\right\}_{s \in S_{0}}$ which meets $G_{s(x)} \cap \operatorname{st}\left(x, \mathcal{W}_{i}\right)$. Hence $\left\{F_{s, i}\right\}_{s \in S_{0}}$ is a discrete and closed family for $X$.

$X$ is collectionwise normal since monotonically normal spaces are collectionwise normal [10]. For every $F_{s, i}$, there exists an open subset $G_{s, i}$ such that $F_{s, i} \subset G_{s, i} \subset G_{s}$ and $\left\{G_{s, i}\right\}_{s \in S_{0}}$ is a discrete family. Let

$$
\mathcal{B}_{i}=\left\{G_{s, i}\right\}_{s \in S_{0}} \cup\left\{\{x\}: x \in I-\bigcup_{s \in S_{0}} G_{s, i}\right\} .
$$

Then $\bigcup_{i \in \mathbb{N}} \mathcal{B}_{i}$ is a $\sigma$-locally finite open cover for $X$ and refines $\left\{G_{s}\right\}_{s \in S}$. Since $X$ is regular, $X$ is paracompact.

Next we shall prove the main theorems in this section.

Theorem 2.7. A space $X$ has a regular base at non-isolated points if and only if $X$ has a strong development at non-isolated points.

Proof. Necessity. Since $X$ has a regular base at non-isolated points, $X$ has a regular base at non-isolated points $\mathcal{B}=\left(\bigcup_{i \in \mathbb{N}} \mathcal{B}_{i}\right) \cup \mathcal{I}(X)$ satisfying Lemma 2.5, where $\mathcal{B}_{i}$ is locally finite at non-isolated points and $\mathcal{B}_{i+1} \cup \mathcal{I}(X)$ refines $\mathcal{B}_{i} \cup \mathcal{I}(X)$ for every $i \in \mathbb{N}$. Put $\mathcal{W}_{i}=\mathcal{B}_{i} \cup \mathcal{I}(X)$. We will show that $\left\{\mathcal{W}_{i}\right\}_{i \in \mathbb{N}}$ is a strong development at non-isolated points for $X$. In fact, for every $x \in X-I$ and each open neighborhood $U$ of $x$, since $\mathcal{B}$ is regular at non-isolated points, there exists an open neighborhood $V \subset U$ of $x$ such that the set of all members of $\mathcal{B}$ that meet both $V$ and $X-U$ is finite. We can denote these finite elements by $B_{1}, B_{2}, \cdots, B_{k}$. Then there exists a $j \in \mathbb{N}$ such that $\mathcal{B}_{j} \cap\left\{B_{i}: i \leq k\right\}=\emptyset$. Hence $\operatorname{st}\left(V, \mathcal{W}_{j}\right) \subset U$.

Sufficiency. Let $\left\{\mathcal{W}_{i}\right\}_{i \in \mathbb{N}}$ be a strong development at non-isolated points for $X$. By Lemma 2.6, $X$ is paracompact. For every $i \in \mathbb{N}$, let $\mathcal{B}_{i}$ be a locally finite open refinement for $\mathcal{W}_{i}$. Without loss of generality, we may assume $\mathcal{B}_{i+1}$ refines $\mathcal{B}_{i}$ for every $i \in \mathbb{N}$. We next prove that $\mathcal{B}=\left(\bigcup_{i \in \mathbb{N}} \mathcal{B}_{i}\right) \cup \mathcal{I}(X)$ is a regular base at non-isolated points for $X$. Obviously $\mathcal{B}$ is a base for $X$. For every $x \in X-I$ and each open neighborhood $U$ of $x$, there exist an open neighborhood $V$ of $x$ and an $i \in \mathbb{N}$ such that $\operatorname{st}\left(V, \mathcal{W}_{i}\right) \subset U$. If $j \geq i$, then

$$
\operatorname{st}\left(V, \mathcal{B}_{j}\right) \subset \operatorname{st}\left(V, \mathcal{B}_{i}\right) \subset \operatorname{st}\left(V, \mathcal{W}_{i}\right) \subset U
$$


However, since each $\mathcal{B}_{j}$ is locally finite, there exists an open neighborhood $W(x)$ of $x$ such that the set of all members of $\bigcup_{j<i} \mathcal{B}_{j}$ that meet $W(x)$ is finite. Let $V_{1}=V \cap W(x)$. Then the set of all members of $\mathcal{B}$ that meet $V_{1}$ and $X-U$ is finite.

Similar to definition 2.2, we say a space $X$ has a development at non-isolated points [7] if there exists a sequence $\left\{\mathcal{W}_{i}\right\}_{i \in \mathbb{N}}$ of open covers for $X$ such that, for every $x \in X-I$ and each open neighborhood $U$ of $x$, there exist an open neighborhood $V$ of $x$ and an $i \in \mathbb{N}$ such that $\operatorname{st}\left(V, \mathcal{W}_{i}\right) \subset U$.

Theorem 2.8. A space $X$ has a regular base at non-isolated points if and only if $X$ is a $T_{2}$-paracompact space with a development at non-isolated points.

Proof. Necessity. By Lemma 2.6 and Theorem 2.7, if $X$ has a regular base at nonisolated points, then $X$ is a $\mathrm{T}_{2}$-paracompact space with a development at non-isolated points.

Sufficiency. Let $\mathrm{X}$ be a $\mathrm{T}_{2}$-paracompact space with a development $\left\{\mathcal{W}_{i}\right\}_{i \in \mathbb{N}}$ at nonisolated points. Since $X$ is a $T_{2}$-paracompact space, there exists a sequence of open covers $\left\{\mathcal{B}_{i}\right\}_{i \in \mathbb{N}}$ for $X$ such that $\mathcal{B}_{i+1}$ is a star refinement of $\mathcal{B}_{i} \wedge \mathcal{W}_{i+1}$ for every $i \in \mathbb{N}$. We next prove that $\left\{\mathcal{B}_{i}\right\}_{i \in \mathbb{N}}$ is a strong development at non-isolated points for $X$. For every $x \in X-I$ and every open neighborhood $U$ of $x$, there exists an $i \in \mathbb{N}$ such that $\operatorname{st}\left(x, \mathcal{W}_{i}\right) \subset U$. Choose a $V \in \mathcal{B}_{i+1}$ such that $x \in V$. Then

$$
\operatorname{st}\left(V, \mathcal{B}_{i+1}\right) \subset \operatorname{st}\left(x, \mathcal{B}_{i}\right) \subset \operatorname{st}\left(x, \mathcal{W}_{i}\right) \subset U .
$$

By Theorem 2.7, $X$ has a regular base at non-isolated points.

Remark We cannot omit the condition " $\mathrm{T}_{2}$ " in Theorem 2.8 , In fact, let $X$ be the finite complement topology on $\mathbb{N}$. Then $X$ is a $T_{1}$-compact and developable space, but it is not a $\mathrm{T}_{2}$-space.

The following corollary is a complement for Lemma 2.5.

Corollary 2.9. A space $X$ has a regular base at non-isolated points if and only if $X$ is a regular space with a development at non-isolated points $\left\{\mathcal{B}_{i} \cup \mathcal{I}(X)\right\}_{i \in \mathbb{N}}$, where $\mathcal{B}_{i}$ is locally finite at non-isolated points for every $i \in \mathbb{N}$.

Proof. Necessity. It is easy to see by the proof of necessity in Theorems 2.7 and 2.8 ,

Sufficiency. Let $X$ be a regular space with a development at non-isolated points $\left\{\mathcal{B}_{i} \cup \mathcal{I}(X)\right\}_{i \in \mathbb{N}}$, where $\mathcal{B}_{i}$ is locally finite at non-isolated points for every $i \in \mathbb{N}$. For each $i \in \mathbb{N}$, let

$$
U_{i}=\left\{x \in X: \mathcal{B}_{i} \text { is locally finite at point } x\right\} .
$$

Then $U_{i}$ is an open subset and $\mathcal{B}_{i}$ is locally finite at each point of $U_{i}$. Since $X-I \subset U_{i}$, $X-U_{i} \subset I$ and $X-U_{i}$ is an open subset for $X$. Hence $U_{i}$ is an open and closed subset for $X$. Thus $\mathcal{B}_{i} \mid U_{i}=\left\{B \cap U_{i}: B \in \mathcal{B}_{i}\right\}$ is an open and locally finite family.

By Theorem 2.8, we only need to prove that $X$ is a paracompact space. In fact, for every open cover $\mathcal{U}$ of $X$ and each $i \in \mathbb{N}$, let

$$
\mathcal{V}_{i}=\left\{B \cap U_{i}: B \in \mathcal{B}_{i} \text { and there exists an } U \in \mathcal{U} \text { such that } B \subset U\right\}
$$

and

$$
V_{i}=\cup \mathcal{V}_{i}
$$


Put

$$
\mathcal{V}=\left(\bigcup_{i \in \mathbb{N}} \mathcal{V}_{i}\right) \cup\{\{x\}: x \in F\}, \text { where } F=\bigcap_{i \in \mathbb{N}}\left(X-V_{i}\right)
$$

Then $\mathcal{V}$ is a cover for $X$ and $F \subset I$. In fact, if $x \in X-I$, then there exists an $U \in \mathcal{U}$ such that $x \in U$. Hence there exists an $n \in \mathbb{N}$ such that $\operatorname{st}\left(x, \mathcal{B}_{n}\right) \subset U$. Fix a $B \in \mathcal{B}_{n}$ such that $x \in B$. Then $B \subset U$ and $x \in B \cap U_{n} \in \mathcal{V}_{n}$. So $x \in V_{n}$. Then $F$ is a closed and discrete subset for $X$. Hence $\mathcal{V}$ is an open $\sigma$-locally finite cover and refines $\mathcal{U}$. By the regularity, $X$ is a paracompact space.

Example 2.10. There exists a non-regular $\mathrm{T}_{2}$-space with a development at non-isolated points.

Let $\mathbb{Q}, \mathbb{P}$ denote the rational numbers and the irrational numbers, respectively. Let $X=\mathbb{R}$ and endow $X$ with the following topology [4]: every point of $\mathbb{P}$ is an isolated point; every point $x \in \mathbb{Q}$ has neighborhoods of the following form:

$$
B(x, n)=\{x\} \cup\{y \in \mathbb{P}:|y-x|<1 / n\}, n \in \mathbb{N} .
$$

Then $X$ is a non-regular $\mathrm{T}_{2}$-space and the isolated points set of $X$ is $\mathbb{P}$. We denote $\mathbb{Q}=\left\{q_{m}: m \in \mathbb{N}\right\}$. For any $n, m \in \mathbb{N}$, let

$$
\mathcal{B}_{n, m}=\left\{B\left(q_{m}, n\right), \mathbb{R}-\left\{q_{m}\right\}\right\},
$$

Then $\mathcal{B}_{n, m}$ is a finite open cover for $X$, and $\operatorname{st}\left(q_{m}, \mathcal{B}_{n, m} \cup \mathcal{I}(X)\right)=B\left(q_{m}, n\right)$. Hence $\left\{\mathcal{B}_{n, m} \cup \mathcal{I}(X)\right\}_{n, m \in \mathbb{N}}$ is a development at non-isolated points for $X$ and $\mathcal{B}_{n, m}$ is locally finite for any $n, m \in \mathbb{N}$.

\section{Metrization Theorems}

In this section we shall discuss the metrization problems on spaces with the properties of bases at non-isolated points.

$X$ is called a perfect space if every open subset of $X$ is an $F_{\sigma}$-set in $X$.

Theorem 3.1. Let $X$ be a space. Then the following are equivalent:

(1) $X$ is metrizable;

(2) $X$ is a perfect space with a regular base at non-isolated points;

(3) $X$ is a perfect space with a strong development at non-isolated points.

Proof. By Theorems 1.1 and 2.7, we only need to prove $(3) \Rightarrow(1)$.

Let $X$ be a perfect space with a strong development at the non-isolated points $\left\{\mathcal{W}_{i}\right\}_{i \in \mathbb{N}}$ of $X$. Then there exists a sequence of open sets $\left\{G_{n}\right\}_{n \in \mathbb{N}}$ such that $X-I=$ $\bigcap_{n=1}^{\infty} G_{n}$. For every $n \in \mathbb{N}$, let $\mathcal{U}_{n}=\left\{G_{n}\right\} \cup\left\{\{x\}: x \in I-G_{n}\right\}$. Then $\left\{\mathcal{U}_{n}\right\}_{n \in \mathbb{N}}$ is a sequence of open covers for $X$. Put $\mathcal{V}_{2 n-1}=\mathcal{W}_{n}$ and $\mathcal{V}_{2 n}=\mathcal{U}_{n}$, for each $n \in \mathbb{N}$. Then $\left\{\mathcal{V}_{n}\right\}_{n \in \mathbb{N}}$ is a strong development for $X$, and $X$ is metrizable by [ 6 , Theorem 5.4.2].

Remark By Example 1.2, we see the condition " $X$ is perfect" in (2) and (3) of Theorem 3.1 cannot be omitted, although clearly it can be replaced with the condition that $I(X)$ is an $F_{\sigma}$-set.

Definition 3.2. Let $\mathcal{B}=\bigcup_{i \in \mathbb{N}} \mathcal{B}_{i}$ be a base for space $X$. $\mathcal{B}$ is called $\sigma$-locally finite at non-isolated points, if for every $i \in \mathbb{N}, \mathcal{B}_{i}$ is locally finite at non-isolated points for $X$. 
Similarly, we can define the notion of spaces with a $\sigma$-discrete base at non-isolated points.

Definition 3.3. Let $\mathcal{B}$ be a family of subsets of $X$. For every $x \in X, \mathcal{B}$ is called hereditarily closure-preserving at $x$ if, for any $H(B) \subset B \in \mathcal{B}, x \in \overline{\cup\{H(B): B \in \mathcal{B}\}}$, then $x \in \cup\{\overline{H(B)}: B \in \mathcal{B}\}$. $\mathcal{B}$ is called a hereditarily closure-preserving collection for $X$ if, for every $x \in X, \mathcal{B}$ is hereditarily closure-preserving at $x$.

It is easy to verify that a collection is hereditarily closure preserving if and only if it is hereditarily closure preserving at non-isolated points.

Lemma 3.4. Let $\mathcal{B}$ be locally finite at non-isolated points for $X$. Then $\mathcal{B}$ is hereditarily closure-preserving.

Proof. Let $\mathcal{B}=\left\{B_{\alpha}: \alpha \in \Gamma\right\}$. For every $\alpha \in \Gamma$, choose $H_{\alpha} \subset B_{\alpha}$. We can assume $x \in X-I$ and denote $\mathcal{H}=\left\{H_{\alpha}\right\}_{\alpha \in \Gamma}$. If $x \in \overline{\cup \mathcal{H}}$, then there exists an open neighborhood $U(x)$ of $x$ such that the set of all members of $\left\{H_{\alpha}\right\}_{\alpha \in \Gamma}$ that meet $U(x)$ is finite because $\left\{H_{\alpha}\right\}_{\alpha \in \Gamma}$ is locally finite at non-isolated points. we denote these finite elements by $H_{\alpha_{1}}, H_{\alpha_{2}}, \cdots, H_{\alpha_{n}}$. Since

$$
\begin{gathered}
\overline{\cup \mathcal{H}}=\overline{\cup\left(\mathcal{H}-\left\{H_{\alpha_{i}}: i \leq n\right\}\right)} \cup \overline{\cup\left\{H_{\alpha_{i}}: i \leq n\right\}}, \text { and } \\
U(x) \cap\left(\cup\left(\mathcal{H}-\left\{H_{\alpha_{i}}: i \leq n\right\}\right)\right)=\emptyset,
\end{gathered}
$$

we have $x \in \overline{\cup\left\{H_{\alpha_{i}}: i \leq n\right\}}$. Hence $x \in \cup \overline{\mathcal{H}}$.

Lemma 3.5. [5] A regular space $X$ is metrizable if and only if $X$ has a $\sigma$-hereditarily closure-preserving base.

Lemma 3.6. Let $X$ be a regular space. Then the following conditions are equivalent:

(1) $X$ is metrizable;

(2) $X$ has a base which is $\sigma$-discrete at non-isolated points;

(3) $X$ has a base which is $\sigma$-locally finite at non-isolated points.

Proof. It is easy to see by Theorem 1.1, Lemmas 3.4 and 3.5

Let $X$ be a topological space and $\tau(X)$ its topology. $g: \mathbb{N} \times X \rightarrow \tau(X)$ is called a $g$-function if, for any $x \in X$ and $n \in \mathbb{N}, x \in g(n, x)$. A space $X$ is called a $\beta$-space [11] if there exists a $g$-function such that, for every $x \in X$ and sequence $\left\{x_{n}\right\}$ in $X$, if $x \in g\left(n, x_{n}\right)$ for each $n \in \mathbb{N}$, then $\left\{x_{n}\right\}$ has a cluster point in $X$. Obviously every developable space is a $\beta$-space.

Theorem 3.7. A space $X$ is metrizable if and only if $X$ is a $\beta$-space with a regular base at non-isolated points.

Proof. We only need to prove the sufficiency. Let $X$ be a $\beta$-space with a regular base at non-isolated points. By Theorem 3.1, it suffices to prove that $I(X)$ is an $F_{\sigma}$-set. Suppose $g$ is a $g$-function satisfying the above definition of $\beta$-spaces. Since $X$ has a regular base at non-isolated points, $X$ has a regular base at non-isolated points $\mathcal{B}=\left(\bigcup_{n \in \mathbb{N}} \mathcal{B}_{n}\right) \cup \mathcal{I}(X)$ satisfying Lemma 2.5, where $\mathcal{B}_{n}$ is locally finite at non-isolated points and $\mathcal{B}_{n+1} \cup \mathcal{I}(X)$ refines $\mathcal{B}_{n} \cup \mathcal{I}(X)$ for each $n \in \mathbb{N}$. For each $n \in \mathbb{N}$ and $x \in X-I$, put

$$
b(n, x)=\cap\left\{B \in \mathcal{B}_{n}: x \in B\right\} .
$$


Then $\{b(n, x)\}_{n \in \mathbb{N}}$ is a local base for $x \in X-I$. For each $n \in \mathbb{N}$, put

$$
\begin{gathered}
h(n, x)=(\cap\{g(i, x): i \leq n\}) \cap b(n, x), x \in X-I ; \\
H_{n}=\cup\{h(n, x): x \in X-I\} .
\end{gathered}
$$

Then $X-I \subset H_{n}$ and $H_{n}$ is an open subset for $X$. We next prove $X-I=\bigcap_{n \in \mathbb{N}} H_{n}$. Let $x \in \bigcap_{n \in \mathbb{N}} H_{n}$. Then there exists some point $x_{n} \in X-I$ such that $x \in h\left(n, x_{n}\right)$ for each $n \in \mathbb{N}$. Since $X$ is a $\beta$-space and $x \in g\left(n, x_{n}\right),\left\{x_{n}\right\}$ has a cluster point in $X$. Let $y$ be a cluster point of $\left\{x_{n}\right\}$. Then $y \in X-I$ and $b(n, y)$ is an open neighborhood of $y$. Without loss of generality, we can assume $x_{n_{i}} \in b(i, y)$ for each $i \in \mathbb{N}$. We will show that $b\left(i, x_{n_{i}}\right) \subset b(i, y)$. If not, choose a point $z \in b\left(i, x_{n_{i}}\right)-b(i, y)$, then there exists a $B \in \mathcal{B}_{i}$ such that $y \in B$ and $z \notin B$. Since $x_{n_{i}} \in b(i, y) \subset B, z \in b\left(i, x_{n_{i}}\right) \subset B$, a contradiction. Hence

$$
x \in \bigcap_{i \in \mathbb{N}} h\left(n_{i}, x_{n_{i}}\right) \subset \bigcap_{i \in \mathbb{N}} h\left(i, x_{n_{i}}\right) \subset \bigcap_{i \in \mathbb{N}} b(i, y)=\{y\},
$$

i.e, $x=y \in X-I$. Thus $X-I=\bigcap_{n \in \mathbb{N}} H_{n}$, and $I$ is an $F_{\sigma}$-set for $X$. By Theorem 3.1 , $X$ is metrizable.

Remark The Stone-Čech compactification $\beta \mathbb{N}$ of $\mathbb{N}$ is a $\beta$-space, but it is not a perfect space [6, Corollary 3.6.15]; Sorgenfrey line is a perfect space, but it is not a $\beta$-space [11, Example 4.4]. Hence, Theorem 3.1 and Theorem 3.7 are independent each other.

\section{Relations with Generalized Metrizable Spaces}

Definition 4.1. 14] Let $X$ be a topological space and let $A$ be a subset of $X$. The discretization of $X$ by $A$ is the space whose topology is generated by the base $\{U$ : $U$ is an open subset of $X\} \cup\{\{x\}: x \in A\}$. It is denoted by $X_{A}$ in [6, Example 5.1.22]. We say that a space $Y$ is a discretization of $X$ if $Y=X_{A}$ for some $A \subset X$.

Theorem 4.2. Let $X$ be a metric space. If $A \subset X$ and $X_{A}$ is the discretization of $X$ by $A$, then $X_{A}$ has a regular base at non-isolated points.

Proof. Since $X$ is a metric space, $X$ has a regular base $\mathcal{B}_{1}$. Let $\mathcal{B}=\mathcal{B}_{1} \cup\{\{x\}: x \in A\}$. Obviously, $\mathcal{B}$ is a regular base at non-isolated points for $X_{A}$.

Remark If a space $X$ with a regular base at non-isolated points, then is it a discretizable space of a metric space? The answer is negative, see Example 4.3. Recall that $X$ is said to have a $G_{\delta^{-}}$diagonal if there exists a sequence $\left\{\mathcal{U}_{n}\right\}_{n \in \mathbb{N}}$ of open covers such that $\{x\}=\bigcap_{n \in \mathbb{N}} \operatorname{st}\left(x, \mathcal{U}_{n}\right)$ for every $x \in X$.

Example 4.3. There exists a space $Y$ having a regular base at non-isolated points. However, $Y$ is not a discretization of a metric space.

Let $X$ be the Michael line in Example 1.2, and denote it by $X_{B}$. Let $X^{*}$ be a copy of $X_{B}$ and $f: X_{B} \rightarrow X^{*}$ a homeomorphic map. Put $Z=X_{B} \bigoplus X^{*}$ and let $g: Z \rightarrow Y$ be a quotient map by identifying $\{x, f(x)\}$ to a point for each $x \in X_{B} \backslash B$ in $Z$. Then $Y$ is a quotient space.

By [16], it is easy to see $Y$ has no $G_{\delta}$-diagonal. Since the discretization of a metric space has a $G_{\delta}$-diagonal, $Y$ is not a discretization of a metric space. We next prove that $Y$ has a regular base at non-isolated points. 
Put $\mathcal{I}=\{\{x\}: x \in B\}$ and let $\mathcal{B}$ be a regular base of $\mathbb{I}$ with the Euclidean topology. Then $\mathcal{B} \cup \mathcal{I}$ is a regular base at non-isolated points for $X_{B}$. Hence $f(\mathcal{B}) \cup f(\mathcal{I})$ is a regular base at non-isolated points for $X^{*}$. Then $\mathcal{G}=\{g(B \cup f(B)): B \in \mathcal{B}\} \cup \mathcal{I} \cup f(\mathcal{I})$ is a regular base at non-isolated points for $Y$.

Indeed, it is easy to see that $\mathcal{G}$ is a base for $Y$. For every $y \in Y-I(Y)$ and each open neighborhood $U$ of $y$ in $Y$, there exists a point $x \in X_{B}$ such that $g(x)=y$. Then $g(f(x))=y$, and $x, f(x) \in g^{-1}(U)$. Since

$$
\mathcal{B}_{0}=\mathcal{B} \cup f(\mathcal{B}) \cup \mathcal{I} \cup f(\mathcal{I})
$$

is a regular base at non-isolated points for $Z$, there exist open neighborhoods $V_{x}, V_{f(x)} \subset$ $g^{-1}(U)$ of $x, f(x)$ in $Z$ respectively such that the set of all members of $\mathcal{B}_{0}$ that meet $V_{x}$ and $Z-g^{-1}(U)$ is finite, and the set of all members of $\mathcal{B}_{0}$ that meet $V_{f(x)}$ and $Z-g^{-1}(U)$ is also finite. Since $f$ is a homeomorphic map, there exists a $B \in \mathcal{B}$ such that $x \in B \subset V_{x}$ and $f(x) \in f(B) \subset V_{f(x)}$. Then $g(x)=y \in g(B \cup f(B)) \subset U$. Since the set of all members of $\mathcal{B}_{0}$ that meet $B \cup f(B)$ and $Z-g^{-1}(U)$ is finite. If $V \in \mathcal{B}_{0}$, then $g^{-1}(g(V))=V$, hence the set of all members of $\mathcal{G}$ that meet $g(B \cup f(B))$ and $Y-U$ is finite. Thus $Y$ has a regular base at non-isolated points.

Definition 4.4. [14] An ortho-base $\mathcal{B}$ for $X$ is a base of $X$ such that either $\cap \mathcal{A}$ is open in $X$ or $\cap \mathcal{A}=\{x\} \notin \mathcal{I}(X)$ and $\mathcal{A}$ is a neighborhood base at $x$ in $X$ for each $\mathcal{A} \subset \mathcal{B}$. A space $X$ is a proto-metrizable space if it is a paracompact space with an ortho-base.

Recall that a space $X$ is called a $\gamma$-space if there exists a $g$-function $g(n, x)$ for $X$ satisfying for each $x \in X$ and sequences $\left\{x_{n}\right\},\left\{y_{n}\right\}$ if $x_{n} \in g\left(n, y_{n}\right)$ and $y_{n} \in g(n, x)$ for each $n \in \mathbb{N}$, then $x_{n} \rightarrow x$.

Theorem 4.5. If a space $X$ has a regular base at non-isolated points, then $X$ is:

(1) a proto-metrizable space, and

(2) a $\gamma$-space.

Proof. (1) By Lemma 2.6 and Theorem 2.7, $X$ is a paracompact space. Also, $X$ has an ortho-base by [7, Theorem 3.4]. Hence $X$ is a proto-metrizable space.

(2) To prove part (2), for each $n \in \mathbb{N}$ and $x \in X$ define a function $g: \mathbb{N} \times X \rightarrow \tau(X)$ as follows: if $x \in I$, then $g(n, x)=\{x\}$; if $x \in X-I$, then $g(n, x)=b(n, x)$, where $b(n, x)$ is the same as in the proof in Theorem 3.7. Then $\{g(n, x)\}_{n \in \mathbb{N}}$ is a decreasing and open neighborhood base of $x$, and if $y \in g(n, x)$, then $g(n, y) \subset g(n, x)$. For each $x \in X$ and sequences $\left\{x_{n}\right\},\left\{y_{n}\right\}$, if $x_{n} \in g\left(n, y_{n}\right)$ and $y_{n} \in g(n, x)$ for each $n \in \mathbb{N}$, then $x_{n} \in g\left(n, y_{n}\right) \subset g(n, x)$, thus $x_{n} \rightarrow x$. Hence $X$ is a $\gamma$-space.

Example 4.6. There exists a proto-metrizable space which has no regular base at nonisolated points.

The proto-metrizable but non- $\gamma$-space described in Section 3 in 9 works.

Remark From the discussion above, it can be seen that spaces with a regular base at non-isolated points are strictly between the discretizations of metrizable spaces and proto-metrizable spaces.

Corollary 4.7. Let $X$ have $a G_{\delta}$-diagonal. Then the following conditions are equivalent:

(1) $X$ is a discretizations of a metrizable space;

(2) $X$ has a regular base at non-isolated points; 
(3) $X$ is a proto-metrizable space.

Proof. By Theorems 4.2 and 4.5, we have $(1) \Rightarrow(2) \Rightarrow(3)$. By [9, Theorem 3.1], it can be obtained $(3) \Rightarrow(1)$.

The condition " $G_{\delta}$-diagonal" cannot be omitted in Corollary 4.7 by Example 4.3 ,

Question 4.8. Under what conditions a proto-metrizable space has a regular base at non-isolated points?

Remark Since a proto-metrizable space is a paracompact space, Theorem 2.8 is an answer for Question 4.8. However, we expect a simpler answer.

Definition 4.9. Let $\mathcal{B}$ be a base of a space $X$. B is point-regular [1] (point-regular at non-isolated points [7, resp.) for $X$, if for each (non-isolated, resp.) point $x \in X$ and $x \in U$ with $U$ open in $X,\{B \in \mathcal{B}: x \in B \not \subset U\}$ is finite.

Obviously, every regular base at non-isolated points is a point-regular base at nonisolated points. In [7, it is proved that a space $X$ has a point-regular base at non-isolated points if and only if $X$ is an open, boundary-compact image of a metric space. On the other hand, a space $X$ is an open, boundary-compact, $s$-image of a metric space if and only if $X$ has a point-countable base which is point-regular at non-isolated points. The following question is posed in [7, Question 5.1]:

Question 4.10. [7, Question 5.1] Let a space X have a point-countable base. If $X$ has a point-regular base at nos-isolated points, is $X$ an open, boundary-compact, s-image of a metric space?

Next, we give an affirmative answer for Question 4.10,

A space $X$ is called metalindelöf if every open cover of $X$ has a point-countable open refinement.

Theorem 4.11. The following are equivalent for a space $X$ :

(1) X has a point-countable base, and has a point-regular base at non-isolated points;

(2) $X$ has a point-countable base which is point-regular at non-isolated points;

(3) $X$ is an open boundary-compact, s-image of a metric space;

(4) $X$ is an open s-image of a metric space, and is an open boundary-compact image of a metric space;

(5) $X$ is a metalindelöf space with a point-regular base at non-isolated points.

Proof. It is proved in [7] that if $\mathcal{P}$ is a point-regular base at non-isolated points for a space $X$, then we can assume that $\mathcal{P}=\bigcup_{n \in \mathbb{N}} \mathcal{P}_{n}$ satisfies the following conditions:

(a) $\mathcal{P}_{n}$ is an open cover and is point-finite at non-isolated points;

(b) $\left\{\mathcal{P}_{n}\right\}$ is a development at non-isolated points for $X$.

$(1) \Rightarrow(2)$. Suppose that $X$ has a point-countable base $\mathcal{B}$, and suppose that $X$ has a point-regular base at non-isolated points $\mathcal{P}$. We can assume that $\mathcal{P}=\bigcup_{n \in \mathbb{N}} \mathcal{P}_{n}$ satisfies the conditions (a) and (b). For each $n \in \mathbb{N}$, put

$$
\begin{aligned}
& \mathcal{B}^{\prime}=\{B \in \mathcal{B}: B \not \subset I(X)\} ; \\
& \mathcal{V}_{n}(B)=\left\{P \in \mathcal{P}_{n}: B \subset P\right\}, \quad \forall B \in \mathcal{B}^{\prime} ;
\end{aligned}
$$




$$
\begin{aligned}
& \hat{P}=\cup\left\{B \in \mathcal{B}^{\prime}: P \in \mathcal{V}_{n}(B)\right\}, \quad \forall P \in \mathcal{P}_{n} ; \\
& \hat{\mathcal{P}}_{n}=\left\{\hat{P}: P \in \mathcal{P}_{n}\right\} .
\end{aligned}
$$

Then $\hat{\mathcal{P}}_{n}$ is point-countable. In fact, if $x \in \hat{P} \in \hat{\mathcal{P}}_{n}$, then there is $B^{\prime} \in \mathcal{B}^{\prime}$ such that $x \in B^{\prime}$ and $P \in \mathcal{V}_{n}\left(B^{\prime}\right)$. Since $\left\{B \in \mathcal{B}^{\prime}: x \in B\right\}$ is countable, and each $\mathcal{V}_{n}(B)$ is finite for each $B \in \mathcal{B}^{\prime}$ by the condition (a), it follows that $\left\{P \in \mathcal{V}_{n}(B): x \in B \in \mathcal{B}^{\prime}\right\}$ is countable.

Put

$$
\hat{\mathcal{P}}=\left(\bigcup_{n \in \mathbb{N}} \hat{\mathcal{P}}_{n}\right) \cup \mathcal{I}(X)
$$

Then $\hat{\mathcal{P}}$ is point-countable. If $x \in U-I$ with $U$ open in $X$, then there is $m \in \mathbb{N}$ such that $x \in \operatorname{st}\left(x, \mathcal{P}_{m}\right) \subset U$ by the condition (b). Take $P \in \mathcal{P}_{m}$ with $x \in P$, then there is $B \in \mathcal{B}^{\prime}$ such that $x \in B \subset P$, thus $P \in \mathcal{V}_{m}(B)$, and $x \in B \subset \hat{P} \subset P \subset U$. So $\hat{\mathcal{P}}$ is a base for $X$. Finally, it is easy to see that $\hat{\mathcal{P}}$ is point-regular at non-isolated points by $\hat{P} \subset P$ for each $P \in \mathcal{P}$. $3.1]$.

$(2) \Rightarrow(3)$ by [7, Corollary, 3.2]. (3) $\Rightarrow(4)$ is obvious. And (4) $\Rightarrow(5)$ by [7, Theorem,

$(5) \Rightarrow(1)$. Let $X$ be a metalindelöf space with a point-regular base at non-isolated points. As in the proof of $(1) \Rightarrow(2)$, there is a sequence $\left\{\mathcal{P}_{n}\right\}$ of open covers of $X$ such that $\left\{\mathcal{P}_{n}\right\}$ is a development at non-isolated points for $X$. For each $n \in \mathbb{N}$, let $\mathcal{B}_{n}$ be a point-countable open refinement of $\mathcal{P}_{n}$. And put

$$
\mathcal{B}=\left(\bigcup_{n \in \mathbb{N}} \mathcal{B}_{n}\right) \cup \mathcal{I}(X)
$$

Then $\mathcal{B}$ is a point-countable base for $X$. In fact, if a non-isolated point $x \in U$ with $U$ open in $X$, then there is $n \in \mathbb{N}$ such that $\operatorname{st}\left(x, \mathcal{P}_{n}\right) \subset U$. Take $B \in \mathcal{B}_{n}$ with $x \in B$, then $x \in B \subset \operatorname{st}\left(x, \mathcal{B}_{n}\right) \subset \operatorname{st}\left(x, \mathcal{P}_{n}\right) \subset U$.

By Theorem 4.11, the following is obtained.

Corollary 4.12. Every space with a regular base at non-isolated points has a pointcountable base.

\section{REFERENCES}

[1] P.S. Aleksandrov, On the metrisation of topological spaces (in Russian). Bull Acad Pol Sci, Sér Sci Math Astron Phys, 8(1960), 135-140.

[2] A.V. Arhangel'skiǔ, W. Just, E.A. Renziczenko and P.J. Szeptycki, Sharp bases and weakly unifrom bases versus point-countable bases, Topology Appl., 100(2000), 39-46.

[3] C.E. Aull, A survey paper on some base axioms, Topology Proc., 3 (1978), 1-36.

[4] H.R. Bennett, D.J. Lutzer, A note on weak $\theta$-refinability, General Topology Appl., 2(1972), 49-54.

[5] D.K. Burke, R. Engelking and D.J. Lutzer, Hereditarily closure-preserving collections and metrization, Proc. Amer. Math. Soc., 51(1975), 483-488.

[6] R. Engelking, General Topology (Revised and completed edition), Heldermann Verlag, Berline, 1989.

[7] F.C. Lin, S. Lin, Uniform covers at non-isolated points, Topology Proc., 32(2008), 259-275.

[8] G. Gruenhage, A note on quasi-metrizability, Canad. J. Math., 29(1977), 360-366.

[9] G. Gruenhage, P. Zenor, Proto-metrizable spaces, Houston. J. Math., 3(1977), 47-53.

[10] R.W. Heath, D.J. Lutzer and P.L. Zenor, Monotonically normal spaces, Trans. Amer. Math. Soc., 178(1973), 481-493. 
[11] R.E. Hodel, Spaces defined by sequences of open covers which guarantee that certain sequences have cluster points, Duke Math. J., 39(1972), 253-263.

[12] R.E. Hodel, Modern metrization theorems, In: K. P. Hart, J. Nagata and J.E. Vaughan, Encyclopedia of General Topology, Elsevier Science Publishers B. V., Amsterdam, 2004, 242-246.

[13] S. Lin, Generalized Metrizable Spaces and Mappings (2nd edition), China Science Press, Beijing, 2007 (in Chinese).

[14] W.F. Lindgren, P.J. Nyikos, Spaces with bases satisfying certain order and intersection properties, Pacific J. Math., 66(1976), 455-476.

[15] E.A. Michael, The product of a normal space and a metric space need not be normal, Bull. Amer. Math. Soc., 69(1963), 375-376.

[16] V. Popov, A perfect map need not preserve a $G_{\delta}$-diagonal, General Topology Appl., 7(1977), 31-33.

[17] M. Sakai, K. Tamano, Y. Yajima, Regular networks for metrizable spaces and Lašnev spaces, Bull. Polish Acad. Math., 46(1998), 121-133.

Fucai Lin: Department of Mathematics and Information Science, Zhangzhou Normal University, Zhangzhou 363000, P. R. China

E-mail address: linfucai2008@yahoo.com.cn

Shou Lin: Institute of Mathematics, Ningde Teachers' College, Ningde, Fujian 352100, P. R. CHINA

E-mail address: linshou@public.ndptt.fj.cn

Heikki Junnila: Department of Mathematics, University of Helsinki, Yliopistonkatu 5, HELSINKI 10, FINLAND

E-mail address: heikki.junnila@helsinki.fi 